\title{
Reliability of hip muscle strength measured in principal and intermediate planes of movement
}

\author{
Basilio AM Goncalves ${ }^{\text {Corresp., } 1,2}$, David J Saxby ${ }^{1,2}$, Adam Kositsky ${ }^{1,2}$, Rod Barrett $^{1,2}$, Laura E Diamond ${ }^{1,2}$ \\ 1 School of Allied Health Sciences, Griffith University, Gold Coast, Queensland, Australia \\ 2 Gold Coast Orthopaedic Research and Education Alliance (GCORE), Menzies Health Institute Queensland, Griffith University, Gold Coast, Queensland, \\ Australia \\ Corresponding Author: Basilio AM Goncalves \\ Email address: b.goncalves@griffith.edu.au
}

Background: Muscle strength testing is widely used in clinical and athletic populations. Commercially available dynamometers are designed to assess strength in three principal planes (sagittal, transverse, frontal). However, the anatomy of the hip suggests muscles may only be recruited submaximally during tasks performed in these principal planes. Objective: To evaluate the inter-session reliability of maximal isometric hip strength in the principal planes and three intermediate planes. Methods: Twenty participants (26.1 \pm 2.7 years, $50 \%$ female) attended two testing sessions $6.2 \pm 1.8$ days apart. Participants completed 3-5 maximal voluntary isometric contractions for hip abduction, adduction, flexion, extension, internal and external rotation measured using a fixed uniaxial load cell (custom rig) and commercial dynamometer (Biodex). Three intermediate hip actions were also tested using the custom rig: extension with abduction, extension with external rotation, and extension with abduction and external rotation. Results: Moderate-toexcellent intraclass correlation coefficients were observed for all principal and intermediate muscle actions using the custom rig (0.72-0.95) and the Biodex (0.85-0.95). The minimum detectable change was also similar between devices (custom rig=11-31\%; Biodex $=9-20 \%$ ). Bland-Altman analysis revealed poor agreement between devices (range between upper and lower limits of agreement $=77-131 \%)$. Conclusions: Although the devices had similar reliability, both devices may lack the sensitivity to detect small changes in hip strength commonly observed following intervention. 
1 Reliability of hip muscle strength measured in principal and intermediate planes of movement 2

3 Basílio AM Gonçalves ${ }^{1,2}$, David J Saxby ${ }^{1,2}$, Adam Kositsky ${ }^{1,2}$, Rod S Barrett ${ }^{1,2}$, Laura E 4 Diamond $^{1,2}$

$6{ }^{1}$ School of Allied Health Sciences, Griffith University, Gold Coast, Queensland 4222, Australia

$7 \quad 2$ Gold Coast Orthopaedic Research and Education Alliance (GCORE), Menzies Health Institute

8 Queensland, Griffith University, Gold Coast, Queensland 4222, Australia

9

10 Corresponding Author:

11 Basílio Gonçalves ${ }^{1,2}$

12 G02 Gold Coast Campus, Griffith University, Queensland 4222, Australia

13 Email address: b.goncalves@griffith.edu.au 


\section{Abstract}

15 Background: Muscle strength testing is widely used in clinical and athletic populations.

16 Commercially available dynamometers are designed to assess strength in three principal planes

17 (sagittal, transverse, frontal). However, the anatomy of the hip suggests muscles may only be recruited submaximally during tasks performed in these principal planes.

19 Objective: To evaluate the inter-session reliability of maximal isometric hip strength in the 20 principal planes and three intermediate planes.

21 Methods: Twenty participants (26.1 \pm 2.7 years, $50 \%$ female) attended two testing sessions $6.2 \pm 1.8$

22 days apart. Participants completed 3-5 maximal voluntary isometric contractions for hip abduction,

23 adduction, flexion, extension, internal and external rotation measured using a fixed uniaxial load

24 cell (custom rig) and commercial dynamometer (Biodex). Three intermediate hip actions were also

25 tested using the custom rig: extension with abduction, extension with external rotation, and

26 extension with abduction and external rotation.

27 Results: Moderate-to-excellent intraclass correlation coefficients were observed for all principal 28 and intermediate muscle actions using the custom rig (0.72-0.95) and the Biodex (0.85-0.95). The 29 minimum detectable change was also similar between devices (custom rig=11-31\%; Biodex=9-

$3020 \%$ ). Bland-Altman analysis revealed poor agreement between devices (range between upper and

31 lower limits of agreement $=77-131 \%$ ).

32 Conclusions: Although the devices had similar reliability, both devices may lack the sensitivity 33 to detect small changes in hip strength commonly observed following intervention. 


\section{Introduction}

35 Deficits in hip muscle strength are common in a broad range of musculoskeletal conditions, 36 including femoroacetabular impingement (FAI) syndrome (Casartelli et al. 2011; Diamond et al. 37 2015), hip osteoarthritis (Arokoski et al. 2002), adductor-related groin pain (Hölmich et al. 1999), 38 knee ligament injuries (Prendergast et al. 2016), chronic ankle instability (McCann et al. 2018), and low back pain (de Sousa et al. 2019). Hip muscle strength is also considered a determinant for 40 athletic performance, as elite players show larger hip strength compared to sub-elite players 41 (Prendergast et al. 2016). Muscle strength is typically assessed using commercially available 42 dynamometers during maximal voluntary muscle contractions and compared with earlier testing sessions, the unaffected limb, and/or a control group (Kierkegaard et al. 2018). Although a motor44 driven dynamometer (MDD) is considered current best practice for measurement of muscle 45 strength (Desmyttere et al. 2019; Martins et al. 2017; Thorborg et al. 2013), these devices are large, 46 expensive, and limited to measurements in three principal planes (i.e. sagittal, frontal, and 47 transverse).

48 Hand-held dynamometers (HHD) are a portable and reliable alternative to an MDD, but 49 measurements are highly dependent on the skill and relative strength of the assessor (Thorborg et al. 2011). Externally-fixed dynamometers minimise influence of the assessor (Desmyttere et al.

51 2019; Martins et al. 2017; Thorborg et al. 2013) and have potential to measure strength in any 52 movement plane, but are currently only used to measure force in principal planes. Given the 53 oblique lines of action the posterior/lateral hip muscles (e.g. piriformis, gluteus medius) (Neumann 54 2010), maximal contraction in planes outside the principal planes may be necessary to generate 55 the highest levels of activation (Buchanan \& Lloyd 1997). Thus, testing maximal strength in the 
56 principal planes alone may not elicit maximal muscle activation, and consequently may be unable

57 to fully evaluate the function of the posterior/lateral hip musculature.

58 Increasing hip strength may effectively restore function in individuals with hip osteoarthritis

59 (Bennell \& Hinman 2011) and adductor-related groin pain (Hölmich et al. 1999), however,

60 strength training appears less effective for individuals with FAI syndrome (Casartelli et al. 2018).

61 This discrepancy in therapeutic effectiveness could relate not only to the complex anatomical

62 structures comprising the hip joint and requirement for control over six degrees of freedom

63 (Neumann 2010), but also to limited understanding of deep hip muscle function (Diamond et al.

64 2016). Pain and reduced range of motion in movements combining hip flexion, hip adduction, and

65 internal rotation are commonly reported in FAI syndrome (Diamond et al. 2015). The hip extensor,

66 abductor, and external rotator muscles have potential to directly oppose motions of impingement

67 (Neumann 2010) and to influence hip joint loading during locomotive tasks (Catelli et al. 2019).

68 As the hip moves to extension, the potential of muscles like piriformis, gluteus medius. and gluteus

69 maximus to produce external rotation torque increases (Delp et al. 1999). Hip rotation angle may

70 also affect the force generation capacity of the larger gluteal muscles (Delp et al. 1999; Ward et

71 al. 2009; Ward et al. 2010). Thus, assessment of muscle force measurements in intermediate planes

72 combining hip extension, abduction, and external rotation may better quantify hip muscle strength

73 in those with FAI syndrome.

74 Given that commercially available dynamometers are limited to measurements of maximal hip

75 strength in the principal planes, we constructed a cost-effective custom rig consisting of a metal

76 frame and load cell in order to measure to measure maximal hip strength in both the principal and

77 intermediate (i.e. oblique to the principal planes) planes with minimal set-up requirements. The

78 primary aim of this study was to evaluate the inter-session reliability of hip muscle force and torque 
79 measurements acquired using the custom rig. The secondary aim was to compare hip flexion,

80 external rotation, and internal rotation torque values and reliability indexes obtained from the

81 custom rig with measurements from an MDD. We hypothesized that hip muscle strength could be

82 reliably assessed in both the principle and intermediate planes using the custom rig, and that the

83 custom rig would have similar measurement error to an MDD.

85 Materials \& Methods

86 Participants

87 Twenty recreationally active adults (age $=26.0 \pm 2.5$ years, range $=23-33$ years, female $=50 \%$,

88 mass $=69.9 \pm 14.0 \mathrm{~kg}$, height $=1.7 \pm 0.1 \mathrm{~m}$, body mass index $=23.1 \pm 3.0 \mathrm{~kg} / \mathrm{m}^{2}$ ) with no history of

89 hip surgery in their lifetime and no history lower limb injury in the past three months were recruited

90 from the university community to participate in this study. Our sample size was determined based

91 on recommendations from Walter et al. (Walter et al. 1998), with requirements for a good level of

92 reliability (an interclass correlation coefficient (ICC) $>0.75$ (Koo \& Li 2016)) and the acquisition

93 of three trials per participant. The study was approved by Griffith University's human research

94 ethics committee (GU Ref No: 2018/700) and all participants provided written informed consent.

95 The study adhered to the Guidelines for Reporting Reliability and Agreement Studies (GRRAS)

96 (Kottner et al. 2011).

97 Procedures

98 Participants attended two testing sessions separated by 3-9 days (6.2 \pm 1.8 days) and were asked to

99 refrain from lower body resistance or unfamiliar exercise in the 24-hours preceding each testing

100 session. Following a 5-minute self-paced warm-up on a cycle ergometer with a 10N resistance,

101 participants completed a sequence of maximal voluntary isometric contractions (MVIC) on (i) a 
102 custom rig - consisting of a uniaxial load-cell (Dacell UU-K500, Korea, capacity $=4903 \mathrm{~N} \pm 1 \%$,

$103 \operatorname{cost}=\sim 400 \mathrm{USD}$ ) with cable and strap fixated to a metal frame that was rigidly coupled to the floor

104 (Figure 1) and (ii) an MDD (System 4 Pro, Biodex Medical Systems, NY, USA) (Figure 2). All

105 participants performed MVICs using their dominant leg (i.e. leg used to kick a ball). Participants

106 rested for 10 minutes when moving between devices to minimise neuromuscular fatigue. Testing

107 device and hip action orders were randomised between participants. The same device and

108 contraction order were used in both testing sessions. Participants completed two submaximal

109 familiarisation contractions (at their own discretion), followed by a minimum of three MVICs for

110 each hip action with standardised verbal encouragement. Further attempts were performed when

111 measured force increased by $>5 \%$ in the third trial compared to the first and/or second trial

112 (Peltonen et al. 2018). Force did not increase by $>5 \%$ after the second attempt in $>65 \%$ of

113 participants and no participant performed more than five attempts. A minimum of 30 seconds and

114 two minutes rest was allowed between contractions and between different muscle actions (e.g.

115 abduction or adduction), respectively (Thorborg et al. 2013).

116 Participants performed six maximal isometric hip strength tasks in the principal planes of

117 movement using a custom rig and an MDD (Figure 2A-D). Additionally, three intermediate muscle

118 actions were tested using the custom rig only: (a) combined hip extension and abduction (E+AB);

119 (b) combined hip extension and external rotation (E+ER); and (c) combined hip extension,

120 abduction, and external rotation $(\mathrm{E}+\mathrm{AB}+\mathrm{ER})$ (Figure $2 \mathrm{E})$. The strength testing body positions were

121 chosen with consideration of the physical constraints of each device. Hip abduction and adduction

122 strength were tested in a supine position using the custom rig and in a side-lying position using the

123 MDD. For both devices, knees were placed in neutral $\left(0^{\circ}\right.$ of flexion $)$ and the hips were positioned

124 in $15^{\circ}$ of abduction and neutral rotation. Hip flexion, extension, and internal and external rotation 
125 strength were tested in the same position for both devices. Hip flexion strength was tested in a

126 supine position with the hip and knee in $45^{\circ}$ and $90^{\circ}$ of flexion, respectively. Hip extension was

127 tested in a prone position with the hip and knee in neutral ( $0^{\circ}$ of flexion). Hip internal and external

128 rotation were tested in a sitting position with the hip and knee in $90^{\circ}$ of flexion. The three

129 intermediate muscle actions $(\mathrm{E}+\mathrm{AB}, \mathrm{E}+\mathrm{ER}$, and $\mathrm{E}+\mathrm{AB}+\mathrm{ER})$ were assessed in a standing position

130 with the hip and knees in neutral ( $0^{\circ}$ of flexion). For $\mathrm{E}+\mathrm{ER}$ and $\mathrm{E}+\mathrm{AB}+\mathrm{ER}$, participants placed

131 their hips at their maximum range of hip external rotation. For $\mathrm{E}+\mathrm{AB}$ and $\mathrm{E}+\mathrm{AB}+\mathrm{ER}$, participants

132 were instructed to produce force at a $45^{\circ}$ angle (midway between pure abduction and pure

133 extension) (Figure 2E). The strap was attached to the distal shank (as close as possible to the lateral

134 malleoli) for hip abduction, adduction, extension, internal rotation, external rotation, and all

135 intermediate plane measurements using the custom rig and for hip internal and external rotation

136 measurements using the MDD. For all other positions, the strap was attached to the distal thigh (as

137 close as possible to the lateral femoral condyle). For measurements acquired using the custom rig,

138 joint angles were confirmed manually with a goniometer (Buchanan \& Lloyd 1997). For hip

139 extension using the custom rig, the angle between the direction of force production (vertical) and

140 the direction of force measurement (30 degrees with respect to vertical) (Figure 2A, right), was

141 controlled for all participants and used to calculate the vertical component of force (Hip extension

142 force $=$ measured force $\left./ \cos \left(30^{\circ}\right)\right)$. For measurements acquired using the MDD, joint angles were

143 confirmed with the device's inbuilt goniometer and the greater trochanter was aligned with the

144 device's axis of rotation. The inbuilt goniometer was calibrated with a digital inclinometer before

145 each task to offset the extra padding in the MDD. For hip abduction/adduction, the axis of rotation

146 of the MDD was aligned with the intersection of a vertical line crossing the anterior superior iliac

147 spine and a horizontal line crossing the greater trochanter (Sugimoto et al. 2014). For hip 
148 internal/external rotation, the axis of rotation was aligned with the centre of the patella (Figure

149 2D). The weight of the test limb was recorded and used to correct for gravity (Buchanan \& Lloyd

150 1997). Measurements acquired in the principal planes using the custom rig were converted to

151 torque $(\mathrm{N} \cdot \mathrm{m})$ by multiplying the measured force $(\mathrm{N})$ by the lever arm $(\mathrm{m})$. The lever arm was

152 measured from the most lateral bony prominence of the greater trochanter to the centre of the

153 attachment point (i.e. strap) for each position. For internal and external rotation, the lever arm was

154 measured from the centre of the patella to the centre of the attachment point.

155

156 Insert Figure 1 here.

157

158

Data processing

159 All data were recorded with Vicon Nexus 2.7.1 software (Vicon, Oxford Metrics Group, UK) at

$1601000 \mathrm{~Hz}$. Data were subsequently low-pass filtered at $6 \mathrm{~Hz}$ using a dual pass $2^{\text {nd }}$ order Butterworth

161 filter. The highest force or torque value from each muscle action was used to determine maximum

162 strength.

163

164 Data analysis

165 We excluded statistical outliers from the analysis following established procedures (Kwak \& Kim

166 2017) and confirmed data normality using the Shapiro-Wilk test (Ghasemi \& Zahediasl 2012). We

167 determined inter-session reliability (Atkinson \& Nevill 1998) using ICC with a 95\% confidence

168 interval, based on a single measurement, absolute-agreement, two-way mixed-effects model (Koo

$169 \&$ Li 2016). ICCs $<0.5$ were interpreted as poor, 0.5-0.74 as moderate, $0.75-0.89$ as good, and $\geq 0.9$

170 as excellent (Koo \& Li 2016). We also estimated absolute reliability (Atkinson \& Nevill 1998) (or 
171 measurement error) using the standard error of measurement (SEM) (the standard deviation of the

172 between-day difference in strength $/ \sqrt{ } 2$ ) (Weir 2005) and minimal detectable change (MDC) based

173 on a $90 \%$ confidence interval $\left(\mathrm{MDC}_{90}=\right.$ the standard deviation of the between-day difference in

174 strength $\times 1.65)($ Weir 2005). Both SEM and MDC were calculated as a percentage (SEM\% and

175 MDC\%) by dividing each absolute value by the grand mean for that strength variable. Reliability

176 and measurement error were calculated for torque and force measurements to ensure the lever arm

177 measurements had no influence on the strength assessment. We assessed the agreement between

178 hip flexion, internal rotation, and external rotation strength using the custom rig and MDD using

179 the Bland-Altman method with data from both testing sessions $(n=40)$ (Bland \& Altman 2010)

180 by plotting the percentage difference in torque between devices ((custom rig-MDD) $/ \mathrm{MDD} \times 100)$

181 against the mean of the two strength measures. Bias and limits of agreement (LoA) were calculated

182 as the mean difference in torque between devices and standard deviation of the mean differences

183 multiplied by 1.96, respectively. The CI for the limits of agreement were calculated as previously

184 reported (Carkeet \& Goh 2018). We did not test for agreement between hip abduction, adduction,

185 or extension given the differences in body position and lever arm between devices, which have

186 been shown to affect absolute force values (Thorborg et al. 2010). All analyses were undertaken

187 using custom scripts in MATLAB R2018a (The MathWorks, Inc., Massachusetts, USA).

188

189 Insert Figure 2 here.

190

191 Results

192 We observed excellent reliability for torque measurements of hip adduction and flexion (ICC =

193 0.92-0.95) and good reliability for all other torque measurements (ICC $=0.77-0.86)$ using the 
194 custom rig, with the exception of external rotation (moderate reliability; ICC $=0.72$ ) $($ Table 1).

195 We observed good-to-excellent reliability for all torque measurements using the MDD (ICC =

196 0.85-0.95) (Table 2). Similarly, we observed good-to-excellent reliability of force measurements

197 in all intermediate planes using the custom rig $(\mathrm{ICC}=0.77-0.90)$ (Table 3). The SEMs ranged

198 from 5-13\% for the custom rig and 4-9\% for the MDD. The MDCs ranged from $11-31 \%$ for the

199 custom rig and 9-20\% for the MDD.

200

201 Insert Table 1 here.

202

203 From the Bland-Altman analysis we observed, on average, a greater torque measured by the MDD

204 for hip flexion (9\%), a greater torque measured by the custom rig for hip external rotation (1\%),

205 and similar values for hip internal rotation. The differences between the upper and lower limits of 206 agreement were 94\% for hip flexion, 131\% for hip internal rotation, and $77 \%$ for hip external 207 rotation (Figure 3).

208

209 Insert Table 2 here.

210

211 Insert Table 3 here.

212

213 Insert Figure 3 here.

214

215 Discussion 
216 This study evaluated the inter-session reliability of a custom rig designed to measure hip muscle

217 strength in both the principal and intermediate planes. We anticipated that if hip strength

218 measurements obtained using the custom rig were shown to be reliable, the custom rig could

219 contribute to improved diagnosis of, and treatment for, strength impairments in clinical and athletic

220 populations. The custom rig showed good-to-excellent reliability for all muscle actions with the

221 exception of hip external rotation, where we observed only moderate reliability. Similar reliability

222 was observed with the MDD. However, we found large measurement error for most measurements

223 on both devices, suggesting small changes in strength may not be detected. Further, the two

224 methods showed poor agreement, suggesting that these devices should not be used

225 interchangeably. Thus, although the custom rig may be used to measure maximal isometric force

226 in principal and intermediate planes, care must be taken when assessing hip external rotation or

227 comparing values with those from a MDD.

228 Mean ICC values for torque measurements in the principal planes using the custom rig 229 demonstrated excellent reliability for hip adduction and flexion good reliability for hip abduction, 230 extension, and internal rotation, and moderate reliability for hip external rotation. These ICCs are 231 similar to those observed for the MDD (0.85-0.95) and reported elsewhere for externally-fixed 232 dynamometer (0.58-0.99) (Bazett-Jones \& Squier 2020; Hickey et al. 2018; Katoh \& Yamasaki 233 2009; Martins et al. 2017; Romero-Franco et al. 2017; Thorborg et al. 2013), hand-held (0.80-

234 0.97) (Thorborg et al. 2010) or other user independent devices (0.76-0.99) (Aramaki et al. 2016;

235 Gonçalves et al. 2021). The large confidence intervals observed for our ICCs across strength 236 measurements (95\%CI 0.41-0.98) may be a function of marginal between-participant variability $237(\mathrm{CV}=20-27 \%)($ Koo \& Li 2016). Conceivably, tighter confidence intervals could be achievable 238 with a more heterogeneous sample. Although large, our confidence intervals were comparable to 
239 previous reports from a range of measurement devices (95\%CI 0.14-1.00) (Charlton et al. 2017;

240 Thorborg et al. 2013; Thorborg et al. 2010), suggesting the custom rig has similar reliability to

241 other commonly used devices. Small differences in reliability between muscle actions are likely

242 explained by the degree of familiarity of participants with these actions (Hopkins et al. 2001).

243 Although our findings suggest the custom rig can reliably assess maximal strength in the principal

244 planes in a healthy population, further research is needed to assess its use in clinical populations.

245 Our force measurements in the intermediate planes showed good reliability (ICC $=0.86-0.88$

246 [95\%CI 0.66-0.95]). Compared to pure extension force (ICC $=0.90[95 \% \mathrm{CI} 0.73-0.97])$, the

247 reliability of hip strength in the intermediate planes did not decline despite the novelty of the tasks.

248 A single study investigated reliability of maximal isometric strength of hip abduction combined

249 with hip external rotation $(\mathrm{n}=20)$ using a belt-fixed HHD (Aramaki et al. 2016). The authors

250 reported higher reliability $(\mathrm{ICC}=0.97-0.98[95 \% \mathrm{CI} 0.94-0.99])$ than we observed for our

251 measurements in the intermediate planes, however, they used a clam testing position, which

252 inherently assesses bilateral hip muscle strength, and only assessed intra-day reliability (Aramaki

253 et al. 2016). However, individuals with hip pathologies often present unilateral symptoms

254 (Agricola et al. 2014), thus there is a need to measure unilateral hip muscle strength when

255 conduction investigations in clinical populations. Although hip muscle strength performed in

256 intermediate planes can be reliably measured, assessments of electromyography (Glaviano \&

257 Bazett-Jones 2020; Lee et al. 2014; McBeth et al. 2012) are still needed to understand if these tasks

258 do indeed recruit the hip-spanning muscles maximally. A future application of the presented device

259 could explore the relationship between hip muscle strength measured in intermediate planes and

260 the presence of hip symptoms and/or dysfunction to identify potential targets for rehabilitation. 
261 The use of ICCs to assess reliability is influenced by between-participant variability (de Vet et al.

262 2006) and has been criticised across the literature (Hopkins et al. 2001; Koo \& Li 2016; Thorborg

263 et al. 2013). Measurement error is more clinically relevant as it defines a cut-off value for

264 meaningful change (Thorborg et al. 2013; Weir 2005). Measurement errors previously reported

265 for hip strength measurements using hand-held (MDC $\approx 7-34 \%$ ) (Charlton et al. 2017; Thorborg

266 et al. 2010), externally-fixed (MDC $\approx 21-69 \%$ ) (Aramaki et al. 2016; Thorborg et al. 2013), or

267 other user-independent (MDC $\approx 14-16 \%$ ) (Gonçalves et al. 2021) dynamometers are in the range of

268 values observed for the custom rig $(\mathrm{MDC}=11-31 \%)$, which suggests the custom rig has similar

269 sensitivity to these devices. Nevertheless, the ranges of measurement error reported in the

270 literature, including our custom rig, suggest that small-to-moderate improvements in hip muscle

271 strength (2-40\%) typically observed following a 6-18 week strength intervention (Blazevich \&

272 Jenkins 2002; Casartelli et al. 2018; Snyder et al. 2009) or strength differences seen, for instance,

273 between individuals with intra-articular hip pathology and controls (Arokoski et al. 2002; Diamond

274 et al. 2015; Kierkegaard et al. 2018), may remain undetected. Thus, other measurements of hip

275 muscle function (e.g. electromyography, musculoskeletal modelling) may be required in

276 combination with hip muscle strength for comprehensive evaluation of hip muscle function.

277 Absolute torque measurements using the custom rig differed, on average, by $0-9 \%$ from the MDD

278 for hip flexion, internal rotation, and external rotation. The large limits of agreement (77-131\%)

279 suggest measurements from both devices could elicit different results and should not be used

280 interchangeably (Bland \& Altman 2010). Our observations are in agreement with previous studies

281 that showed large disagreement between measurements from hand-held dynamometers and MDD

282 (Bazett-Jones \& Squier 2020; Martins et al. 2017). However, strong relationships ( $\mathrm{r}=0.86$ to 1.00$)$

283 have been found between load-cell and MDD force measures when both devices have 
284 simultaneously acquired data from the same trial (i.e., both the load cell and the MDD were

285 attached to the participant) (Romero-Franco et al. 2017). Nevertheless, when the load cell

286 measurement error (SEM) was compared between days, the researchers showed similar results to

287 those reported here (3.9 to 16.3\%) (Romero-Franco et al. 2017) This suggest differences found

288 between devices found in ours and other studies (Bazett-Jones \& Squier 2020; Martins et al. 2017)

289 could be partly attributed physiological variation that is expected when measuring human subjects

290 (e.g. fatigue, motivation, task familiarity) (Caruso et al. 2012).

291 Measurement errors for the custom rig $(\mathrm{MDC}=11-31 \%)$ were similar to the MDD $(\mathrm{MDC}=9$ -

292 20\%), with the exception of hip extension (20\% vs 9\%). The MDD was attached at a short lever

293 arm for hip extension whereas the load cell on the custom rig was attached at a long lever arm.

294 The latter position could have allowed participants to recruit both the hip extensor and knee flexor

295 muscles, as suggested by results of previous studies where a similar set-up was used for knee flexor

296 strength testing (Askling et al. 2006; Hickey et al. 2018; Yanagisawa \& Fukutani 2020). Despite

297 asking participants to avoid knee flexion and rigorously inspecting for knee flexion during hip

298 extension strength assessments using the custom rig, participants may not have used the same

299 strategy (i.e. hip or knee dominant) with both devices, which could explain differences in

300 measurement error. Thus, when using the custom rig, the ideal position to test hip extension

301 strength still requires further investigation. Additionally, limits of agreement were generally larger

302 than the measurement error of either device, which suggests that differences between devices may

303 be caused by true differences in task performance rather than measurement error from either

304 device. Nevertheless, the two devices appear to be measuring muscle strength originating from

305 different movement strategies, thus comparisons between datasets from different devices should

306 be made with caution. 
307 We have uniquely demonstrated that unilateral maximal isometric hip strength can be measured in

308 intermediate planes with good reliability using a simple, inexpensive, and easily replicable

309 experimental set-up. The load cell used ( $\$ 400$ USD) is cheaper than a hand-held dynamometer

310 (Ishøi et al. 2019; Zhang et al. 2018) or other commercially available devices (e.g. The GroinBar

311 / ForceFrame) (Desmyttere et al. 2019; O'Brien et al. 2019) currently used to assess hip strength.

312 However, the presented set-up was developed in a specialised biomechanics laboratory and some

313 refinements would be required for it to be used in a clinical or athletic setting. A possible solution

314 would be to fixate the load cell to an appropriately stable and supportive object (e.g. door or squat

315 rack) as used previously (Hickey et al. 2018). Additionally, the recording devices used in our

316 experiment can be substituted by an affordable and portable hardware and software ( $\sim$ 100USD,

317 e.g., USB-200 Series, MC measurement computing, Norton, MA, USA). This modified set-up

318 would also allow for force to be acquired at other joints, though reliability would first need to be

319 established.

320 This study has some limitations that warrant consideration. Only two testing sessions were

321 performed, which may explain the moderate reliability of some strength measurements (Hopkins

322 et al. 2001; Koo \& Li 2016). Further, only healthy young adults (23-33 years) were included, and

323 it is unclear whether these results can be extrapolated to other populations with lower-limb

324 musculoskeletal conditions. We also only tested one position for each strength tasks and as

325 previously suggested, body position can influence reliability measurements (Bazett-Jones \&

326 Squier 2020; Thorborg et al. 2010) as well as muscle recruitment (Glaviano \& Bazett-Jones 2020;

327 Yanagisawa \& Fukutani 2020). Thus, it remains unclear whether other positions could be more

328 reliable or elicit higher levels of recruitment from the posterior/lateral hip muscles. Finally, we

329 only compared the validity of the custom rig for three strength tasks (hip flexion, hip internal 
330 rotation, and hip external rotation) because these tasks had a similar set-up between devices.

331 However, since these tasks showed poor agreement between devices, we anticipate the remaining

332 tasks would show similar results.

333

334 Conclusions

335 Good-to-excellent inter-session reliability was generally observed for maximal hip strength 336 measurements performed in principal and intermediate planes using a custom rig. The 337 measurement error associated with the custom rig was similar to that of an MDD, suggesting the 338 custom rig may have utility in studies where large effect sizes are expected, though both devices 339 may lack the sensitivity required to detect small changes in hip strength $(<11-31 \%)$ commonly 340 observed following intervention.

341

\section{Acknowledgements}

343 The authors would like to thank all the volunteers who participated in the study and Alastair Quinn

344 (Griffith University) for his contribution to the manuscript. 


\section{References}

346 Agricola R, Waarsing JH, Thomas GE, Carr AJ, Reijman M, Bierma-Zeinstra SMA, Glyn-Jones

347 S, Weinans H, and Arden NK. 2014. Cam impingement: Defining the presence of a cam

348 deformity by the alpha angle. Data from the CHECK cohort and Chingford cohort.

$349 \quad$ Osteoarthritis and Cartilage 22:218-225. 10.1016/j.joca.2013.11.007

350 Aramaki H, Katoh M, Hiiragi Y, Kawasaki T, Kurihara T, and Ohmi Y. 2016. Validity and reliability of isometric muscle strength measurements of hip abduction and abduction with external hip rotation in a bent-hip position using a handheld dynamometer with a belt. Journal of Physical Therapy Science 28:2123-2127. 10.1589/jpts.28.2123

Arokoski MH, Arokoski JP, Haara M, Kankaanpaa M, Vesterinen M, Niemitukia LH, and Helminen HJ. 2002. Hip muscle strength and muscle cross sectional area in men with and

Askling C, Saartok T, and Thorstensson A. 2006. Type of acute hamstring strain affects flexibility, strength, and time to return to pre-injury level. British Journal of Sports Medicine 40:40-44. 10.1136/bjsm.2005.018879

360

361

362

363

364

365 without hip osteoarthritis. J Rheumatol 29:2185-2195. 0315162X-29-2185 [pii]

Atkinson G, and Nevill AM. 1998. Statistical methods for assessing measurement error (reliability) in variables relevant to sports medicine. Sports Medicine 26:217-238. $10.2165 / 00007256-199826040-00002$

Bazett-Jones DM, and Squier K. 2020. Measurement properties of hip strength measured by handheld dynamometry: Reliability and validity across the range of motion. Physical Therapy in Sport 42:100-106. 10.1016/j.ptsp.2020.01.005 
366 Bennell KL, and Hinman RS. 2011. A review of the clinical evidence for exercise in

367

368

369

370

371

372

373

374

375

376

377

378

379

380

381

382

383

384

385

386

387

388 osteoarthritis of the hip and knee. Journal of Science and Medicine in Sport 14:4-9. 10.1016/j.jsams.2010.08.002

Bland JM, and Altman DG. 2010. Statistical methods for assessing agreement between two methods of clinical measurement. Pergamon. p 931-936.

Blazevich AJ, and Jenkins DG. 2002. Effect of the movement speed of resistance training exercises on sprint and strength performance in concurrently training elite junior sprinters. Journal of Sports Sciences 20:981-990. 10.1080/026404102321011742

Buchanan TS, and Lloyd DG. 1997. Muscle activation at the human knee during isometric flexion-extension and varus-valgus loads. Journal of Orthopaedic Research 15:11-17. 10.1002/jor.1100150103

Carkeet A, and Goh YT. 2018. Confidence and coverage for Bland-Altman limits of agreement and their approximate confidence intervals. Statistical Methods in Medical Research 27:1559-1574. 10.1177/0962280216665419

Caruso JF, Brown LE, and Tufano JJ. 2012. The reproducibility of isokinetic dynamometry data. Isokinetics and Exercise Science 20:239-253. 10.3233/IES-2012-0477

Casartelli NC, Bizzini M, Maffiuletti NA, Sutter R, Pfirrmann CW, Leunig M, and Naal FD. 2018. Exercise therapy for the management of femoroacetabular impingement syndrome: preliminary results of clinical responsiveness. Arthritis Care \& Research 41. $10.1002 /$ acr. 23728

Casartelli NC, Maffiuletti NA, Item-Glatthorn JF, Staehli S, Bizzini M, Impellizzeri FM, and Leunig M. 2011. Hip muscle weakness in patients with symptomatic femoroacetabular impingement. Osteoarthritis and Cartilage 19:816-821. 10.1016/j.joca.2011.04.001

PeerJ reviewing PDF | (2020:10:54315:2:0:NEW 29 Apr 2021) 
389 Catelli DS, Ng KCG, Kowalski E, Beaulé PE, and Lamontagne M. 2019. Modified gait patterns

390 due to cam FAI syndrome remain unchanged after surgery. Gait and Posture 72:135-141.

$391 \quad$ 10.1016/j.gaitpost.2019.06.003

392 Charlton PC, Mentiplay BF, Grimaldi A, Pua YH, and Clark RA. 2017. The reliability of a 393 maximal isometric hip strength and simultaneous surface EMG screening protocol in

394

395

396

397

398

399

400

401

402

403

404

405

406

407

408

409

410 elite, junior rugby league athletes. Journal of Science and Medicine in Sport 20:139-145. 10.1016/j.jsams.2016.06.008

de Sousa CS, de Jesus FLA, Machado MB, Ferreira G, Ayres IGT, de Aquino LM, Fukuda TY, and Gomes-Neto M. 2019. Lower limb muscle strength in patients with low back pain: A systematic review and meta-analysis. Journal of Musculoskeletal Neuronal Interactions 19:69-78.

de Vet HCW, Terwee CB, Knol DL, and Bouter LM. 2006. When to use agreement versus reliability measures. Journal of Clinical Epidemiology 59:1033-1039. 10.1016/j.jclinepi.2005.10.015

Delp SL, Hess WE, Hungerford DS, and Jones LC. 1999. Variation of rotation moment arms with hip flexion. Journal of Biomechanics 32:493-501. 10.1016/S0021-9290(99)00032-9

Desmyttere G, Gaudet S, and Begon M. 2019. Test-retest reliability of a hip strength assessment system in varsity soccer players. Physical Therapy in Sport. 10.1016/j.ptsp.2019.03.013

Diamond LE, Dobson FL, Bennell KL, Wrigley TV, Hodges PW, and Hinman RS. 2015. Physical impairments and activity limitations in people with femoroacetabular impingement: a systematic review. British Journal of Sports Medicine 49:230-242. 10.1136/bjsports-2013-093340 
411 Diamond LE, Wrigley TV, Hinman RS, Hodges PW, O'Donnell J, Takla A, and Bennell KL.

412 2016. Isometric and isokinetic hip strength and agonist/antagonist ratios in symptomatic

413 femoroacetabular impingement. Journal of Science and Medicine in Sport 19:696-701.

$414 \quad 10.1016 /$ j.jsams.2015.10.002

415 Ghasemi A, and Zahediasl S. 2012. Normality tests for statistical analysis: A guide for non416 statisticians. International journal of endocrinology and metabolism 10:486-489. 10.5812/ijem.3505

418 Glaviano NR, and Bazett-Jones DM. 2020. The Influence of Sagittal Plane Hip Position on 419 Lower-Extremity Muscle Activity and Torque Output. J Sport Rehabil:1-9. $420 \quad 10.1123 /$ jsr.2020-0268

421 Gonçalves BAM, Mesquita RNO, Tavares F, Brito J, Correia P, Santos P, and Mil-Homens P. 422 2021. A New Portable Device to Reliably Measure Maximal Strength and Rate of Force Development of Hip Adduction and Abduction. Journal of Strength and Conditioning Research Publish Ahead of Print. 10.1519/jsc.0000000000003872

Hickey JT, Hickey PF, Maniar N, Timmins RG, Williams MD, Pitcher CA, and Opar DA. 2018. A Novel Apparatus to Measure Knee Flexor Strength During Various Hamstring Exercises: A Reliability and Retrospective Injury Study. Journal of Orthopaedic and Sports Physical Therapy 48:72-79. 10.2519/jospt.2018.7634 Krogsgaarda K. 1999. Effectiveness of active physical training as treatment for longstanding adductor-related groin pain in athletes: Randomised trial. Lancet 353:439-443. 10.1016/S0140-6736(98)03340-6 
433 Hopkins WG, Schabort, and Hawley. 2001. Reliability of power in physical performance tests. $434 \quad$ Sports Medicine 31:211-234 224p.

435 Ishøi L, Hölmich P, and Thorborg K. 2019. Measures of hip muscle strength and rate of force 436

Katoh M, and Yamasaki H. 2009. Test-Retest Reliability of Isometric Leg Muscle Strength

Measurements Made Using a Hand-Held Dynamometer Restrained by a Belt: development using a fixated handheld dynamometer: intra-tester intra-day reliability of a clinical set-up. International journal of sports physical therapy 14:715-723.

$10.26603 /$ ijspt20190715 Comparisons during and between Sessions. Journal of Physical Therapy Science 21:239243. $10.1589 /$ jpts. 21.239

Kierkegaard S, Mechlenburg I, Lund B, Rømer L, Søballe K, and Dalgas U. 2018. Is hip muscle strength normalised in patients with femoroacetabular impingement syndrome one year after surgery?: Results from the HAFAI cohort. Journal of Science and Medicine in Sport 22:413-419. 10.1016/j.jsams.2018.10.004

Koo TK, and Li MY. 2016. A Guideline of Selecting and Reporting Intraclass Correlation Coefficients for Reliability Research. Journal of Chiropractic Medicine 15:155-163. 10.1016/j.jcm.2016.02.012

Kottner J, Audigé L, Brorson S, Donner A, Gajewski BJ, Hróbjartsson A, Roberts C, Shoukri M, and Streiner DL. 2011. Guidelines for reporting reliability and agreement studies (GRRAS) were proposed. Journal of Clinical Epidemiology 64:96-106. 10.1016/j.jclinepi.2010.03.002

Kwak SK, and Kim JH. 2017. Statistical data preparation: Management of missing values and outliers. Korean Journal of Anesthesiology 70:407-411. 10.4097/kjae.2017.70.4.407 
456 Lee JH, Cynn HS, Kwon OY, Yi CH, Yoon TL, Choi WJ, and Choi SA. 2014. Different hip

457

458

459

460

461

462

463

464

465

466

467

468

469

470

471

472

473

474

475

476

477

rotations influence hip abductor muscles activity during isometric side-lying hip abduction in subjects with gluteus medius weakness. Journal of Electromyography and Kinesiology 24:318-324. 10.1016/j.jelekin.2014.01.008

Martins J, da Silva JR, da S, Barbosa MR, and Bevilaqua-Grossi D. 2017. Reliability and Validity of the Belt-Stabilized Handheld Dynamometer in Hip- and Knee-Strength Tests. Journal of Athletic Training 52:809-819. 10.4085/1062-6050-52.6.04

McBeth JM, Earl-Boehm JE, Cobb SC, and Huddleston WE. 2012. Hip muscle activity during 3 side-lying hip-strengthening exercises in distance runners. Journal of Athletic Training 47:15-23. 10.4085/1062-6050-47.1.15

McCann RS, Bolding BA, Terada M, Kosik KB, Crossett ID, and Gribble PA. 2018. Isometric hip strength and dynamic stability of individuals with chronic ankle instability. Journal of Athletic Training 53:672-678. 10.4085/1062-6050-238-17

Neumann DA. 2010. Kinesiology of the Hip: A Focus on Muscular Actions. Journal of Orthopaedic \& Sports Physical Therapy 40:82-94. 10.2519/jospt.2010.3025

O'Brien M, Bourne M, Heerey J, Timmins RG, and Pizzari T. 2019. A novel device to assess hip strength: Concurrent validity and normative values in male athletes. Physical Therapy in Sport 35:63-68. 10.1016/j.ptsp.2018.11.006

Peltonen H, Walker S, Hackney AC, Avela J, and Häkkinen K. 2018. Increased rate of force development during periodized maximum strength and power training is highly individual. European Journal of Applied Physiology 118:1033-1042. 10.1007/s00421018-3836-9 
478 Prendergast N, Hopper D, Finucane M, and Grisbrook TL. 2016. Hip adduction and abduction 479 strength profiles in elite, sub-elite and amateur Australian footballers. J Sci Med Sport 480 19:766-770. 10.1016/j.jsams.2015.12.005

481 Romero-Franco N, Jiménez-Reyes P, and Montaño-Munuera JA. 2017. Validity and reliability of 482 a low-cost digital dynamometer for measuring isometric strength of lower limb. Journal of Sports Sciences 35:2179-2184. 10.1080/02640414.2016.1260152

484 Snyder KR, Earl JE, O'Connor KM, and Ebersole KT. 2009. Resistance training is accompanied 485 by increases in hip strength and changes in lower extremity biomechanics during running.

Sugimoto D, Mattacola CG, Mullineaux DR, G TP, and Hewett TE. 2014. Comparison of 488 Isokinetic Hip Abduction and Adduction Peak Torques and Ratio Between Sexes. Clin J Sport Med 24:422-428. 10.1097/JSM.0000000000000059.Comparison

Thorborg K, Bandholm T, and Hölmich P. 2013. Hip- and knee-strength assessments using a 491 hand-held dynamometer with external belt-fixation are inter-tester reliable. Knee Surgery, Sports Traumatology, Arthroscopy 21:550-555. 10.1007/s00167-012-2115-2

493

494

495

496

497 498 499 Clinical Biomechanics 24:26-34. 10.1016/j.clinbiomech.2008.09.009

Thorborg K, Bandholm T, Schick M, Jensen J, and Hölmich P. 2011. Hip strength assessment using handheld dynamometry is subject to intertester bias when testers are of different sex and strength. Scandinavian Journal of Medicine and Science in Sports 23:487-493. 10.1111/j.1600-0838.2011.01405.x

Thorborg K, Petersen J, Magnusson SP, and Hölmich P. 2010. Clinical assessment of hip strength using a hand-held dynamometer is reliable. Scandinavian Journal of Medicine and Science in Sports 20:493-501. 10.1111/j.1600-0838.2009.00958.x 
500 Walter SD, Eliasziw M, and Donner A. 1998. Sample size and optimal designs for reliability

$501 \quad$ studies. Statistics in Medicine 17:101-110. 10.1002/(SICI)1097-

502 0258(19980115)17:1<101::AID-SIM727>3.0.CO;2-E

503 Ward SR, Eng CM, Smallwood LH, and Lieber RL. 2009. Are current measurements of lower 504 extremity muscle architecture accurate? Clinical Orthopaedics and Related Research 505 467:1074-1082. 10.1007/s11999-008-0594-8

506 Ward SR, Winters TM, and Blemker SS. 2010. The architectural design of the gluteal muscle group: Implicati ons for movement and rehabilitation. Journal of Orthopaedic and Sports Physical Therapy 40:95-102. 10.2519/jospt.2010.3302

509 Weir JP. 2005. Quantifying Test-Rest Reliability Using the Intraclass Correlation Coefficient $510 \quad$ and the SEM. J Str Cond Res 19:231-240.

511 Yanagisawa O, and Fukutani A. 2020. Muscle Recruitment Pattern of the Hamstring Muscles in 512 Hip Extension and Knee Flexion Exercises. Journal of Human Kinetics 72:51-59. 10.2478/hukin-2019-0124

514 Zhang ZJ, Lee WC, Ng GYF, and Fu SN. 2018. Isometric strength of the hip abductors and 515 external rotators in athletes with and without patellar tendinopathy. European Journal of Applied Physiology 118:1635-1640. 10.1007/s00421-018-3896-x 
Figure 1

Schematic representation of the metal frame used to fixate the load cell.

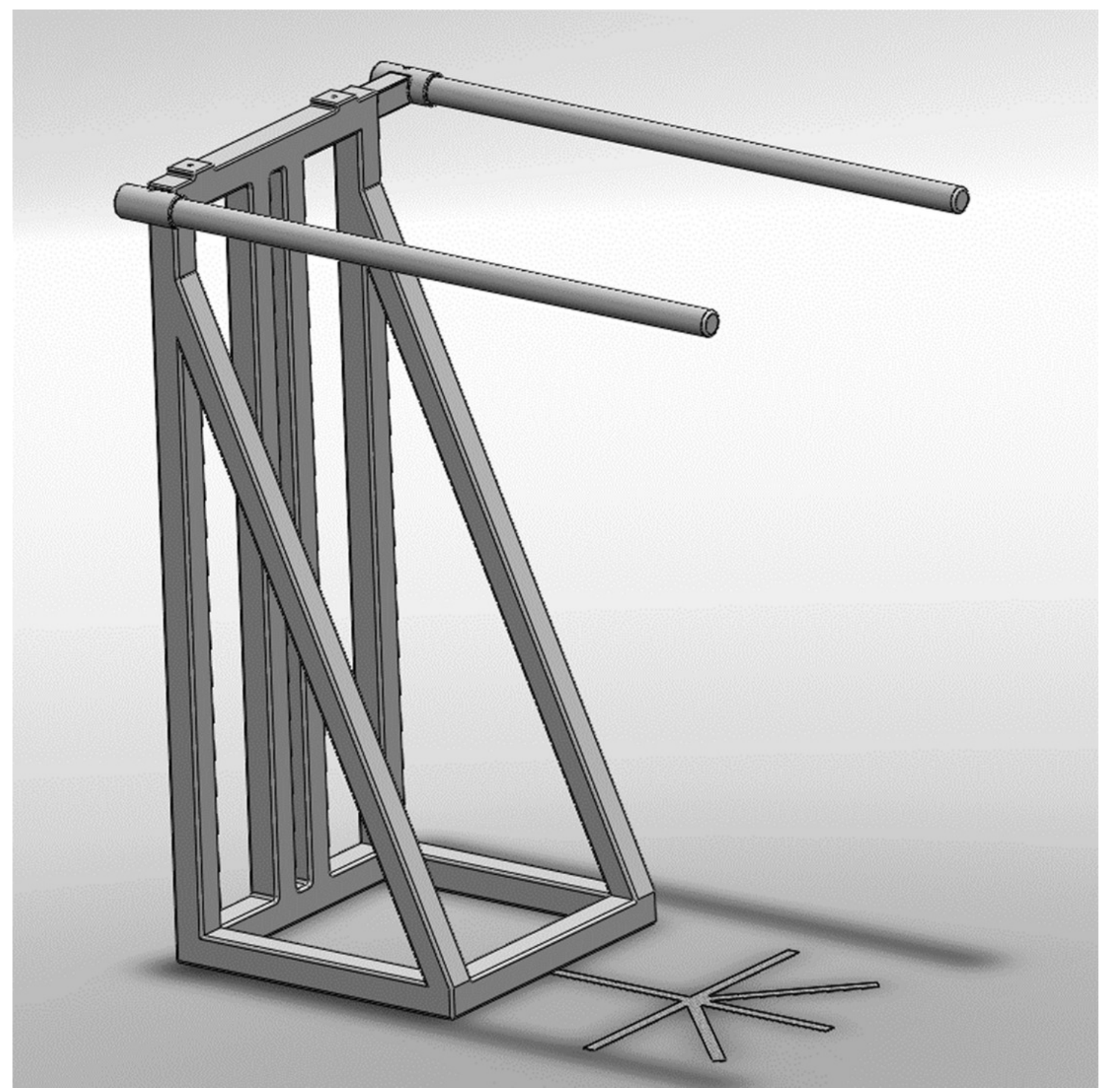




\section{Figure 2}

Hip muscle strength testing positions using a motor-driven dynamometer (MDD) and a custom rig.

(A) hip extension using the MDD (left) and custom rig (right); (B) hip flexion using the MDD (left) and the custom rig (right); (C) hip abduction/adduction using the MDD (left) and the custom rig (right); (D) hip internal/external rotation using the MDD (left) and the custom rig (right). 

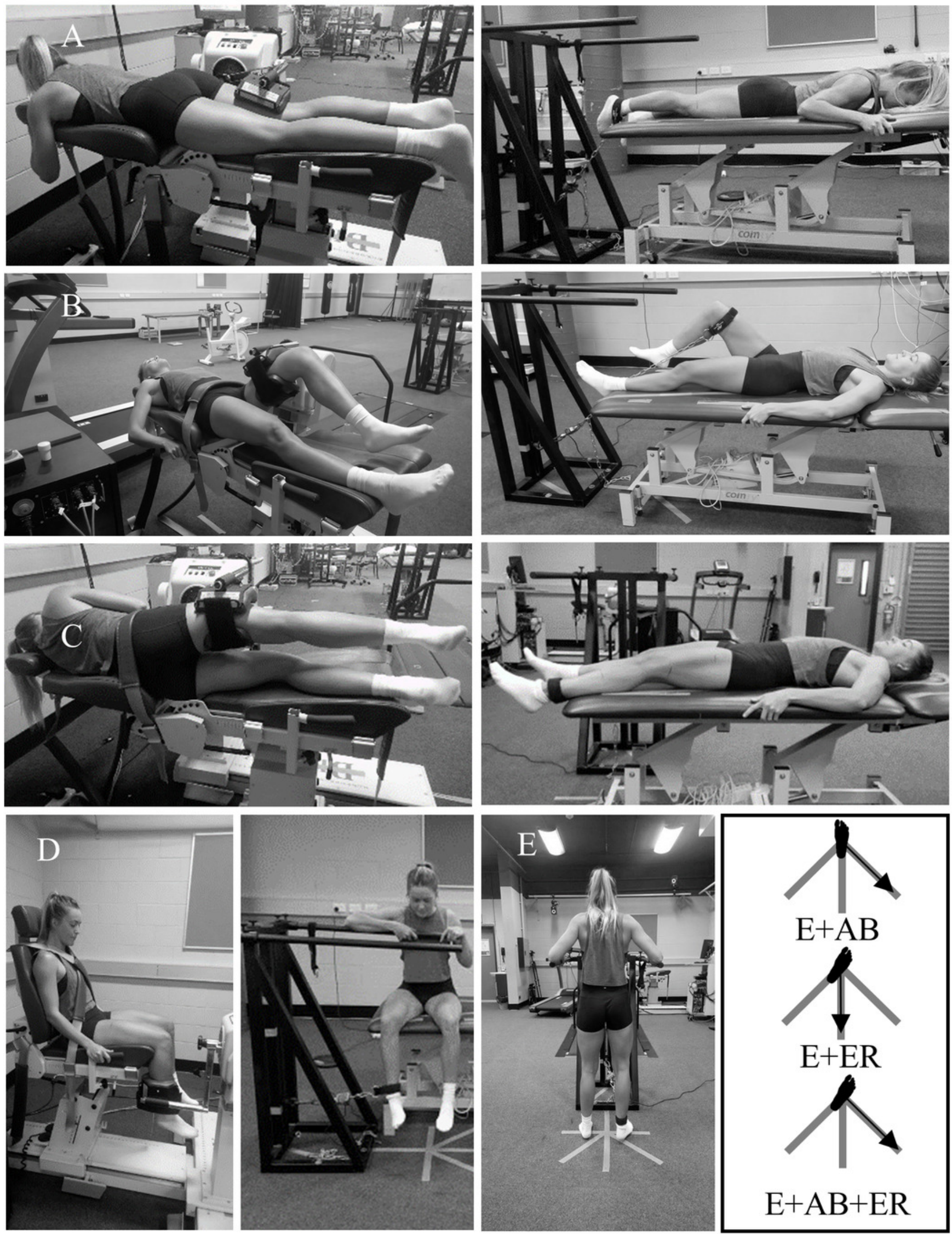
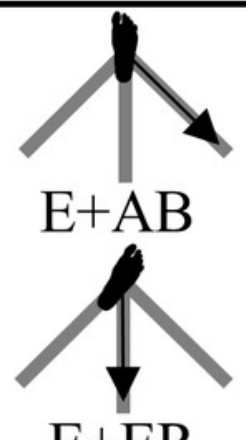

$\mathrm{E}+\mathrm{ER}$

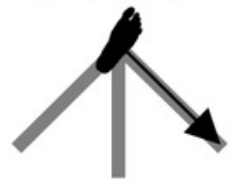

$\mathrm{E}+\mathrm{AB}+\mathrm{ER}$ 
Figure 3

Bland-Altman analysis of hip strength measurements.

Hip flexion (left), hip internal rotation (middle), and hip external rotation (right), showing differences in mean torque values between devices (custom rig) - motor-driven dynamometer (MDD); solid line), and upper (uLoA) and lower (ILOA) limits of agreement (dashed lines) $(n=40)$.

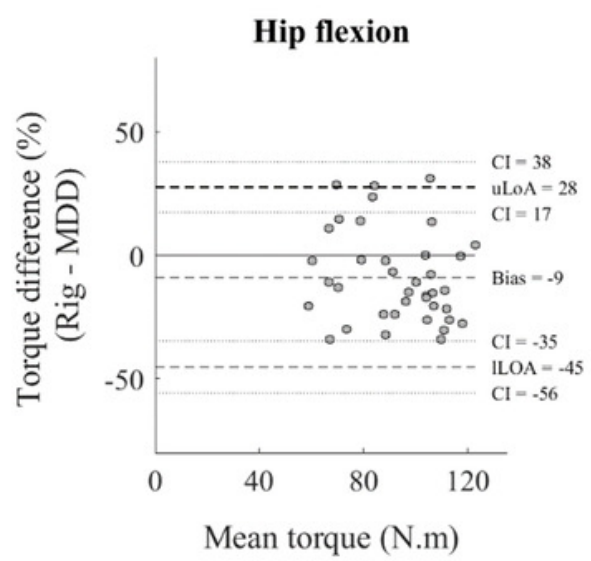

Mean torque (N.m)

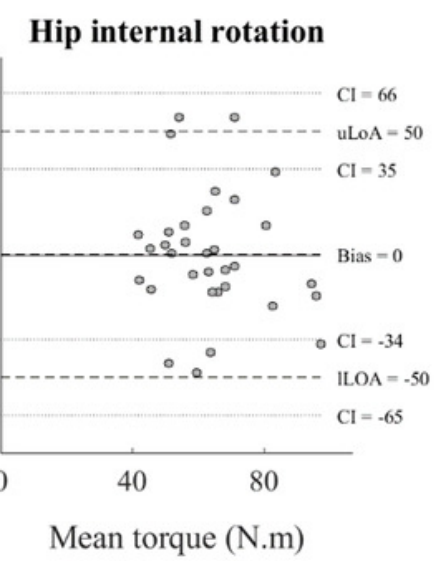

Hip external rotation

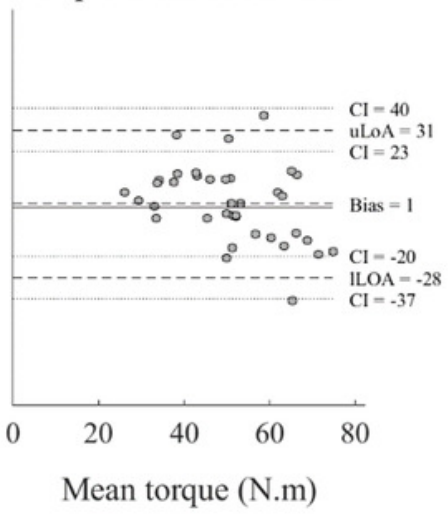




\section{Table 1 (on next page)}

Reliability metrics for hip torque measurements using a custom rig.

Values are mean \pm standard deviation unless otherwise indicated; ICC $=$ intraclass

correlation coefficient; $\mathrm{Cl}=$ confidence interval; SEM = standard error of measurement; MDC

$=$ minimal detectable change; $\mathrm{N} \cdot \mathrm{m}=$ newton.metre; $\mathrm{N}=$ newton; $\mathrm{E}=$ extension; $\mathrm{AB}=$ abduction; $E R$ = external rotation; 
1 Table 1. Reliability metrics for hip torque measurements using a custom rig.

\begin{tabular}{|c|c|c|c|c|c|c|c|}
\hline & Session 1 & Session 2 & ICC (95\%CI) & SEM (95\%CI) & SEM & $\begin{array}{l}\text { MDC } \\
(95 \% C I)\end{array}$ & MDC \\
\hline & $(\mathrm{N} \cdot \mathrm{m})$ & $(\mathrm{N} \cdot \mathrm{m})$ & & $(\mathrm{N} \cdot \mathrm{m})$ & $\%$ & $(\mathrm{~N} \cdot \mathrm{m})$ & $\%$ \\
\hline Abduction (n=20) & $95 \pm 21$ & $99 \pm 24$ & $0.77(0.51-0.90)$ & $11(8-16)$ & 11 & $25(19-37)$ & 26 \\
\hline Adduction $(n=20)$ & $124 \pm 29$ & $126 \pm 26$ & $0.92(0.79-0.97)$ & $8(6-12)$ & 6 & $19(14-28)$ & 15 \\
\hline Extension $(n=18)$ & $226 \pm 54$ & $210 \pm 52$ & $0.85(0.57-0.95)$ & $19(14-29)$ & 9 & $44(32-68)$ & 20 \\
\hline Flexion $(n=17)$ & $87 \pm 17$ & $88 \pm 17$ & $0.95(0.84-0.98)$ & $4(3-7)$ & 5 & $9(7-15)$ & 11 \\
\hline Internal rotation $(n=20)$ & $65 \pm 19$ & $65 \pm 17$ & $0.86(0.66-0.94)$ & $7(5-10)$ & 11 & $16(12-24)$ & 25 \\
\hline $\begin{array}{l}\text { External } \\
(\mathbf{n}=\mathbf{2 0})\end{array}$ & $48 \pm 11$ & $50 \pm 13$ & $0.72(0.41-0.88)$ & $6(5-10)$ & 13 & $15(11-22)$ & 31 \\
\hline
\end{tabular}

2

3 Values are mean \pm standard deviation unless otherwise indicated; $\mathrm{ICC}=$ intraclass correlation coefficient; $\mathrm{CI}=$ confidence interval;

$4 \mathrm{SEM}=$ standard error of measurement $\mathrm{MDC}=$ minimal detectable change; $\mathrm{N} \cdot \mathrm{m}=$ newton.metre $\mathrm{N}=$ newton; $\mathrm{E}=$ extension; $\mathrm{AB}=$

5 abduction; $\mathrm{ER}=$ external rotation; 


\section{Table 2 (on next page)}

Reliability metrics for hip torque measurements using motor-driven dynamometer.

Values are mean \pm standard deviation unless otherwise indicated; ICC $=$ intraclass

correlation coefficient; $\mathrm{Cl}=$ confidence interval; SEM = standard error of measurement; MDC

$=$ minimal detectable change; $\mathrm{N} \cdot \mathrm{m}=$ newton.metre; $\mathrm{N}=$ newton; $\mathrm{E}=$ extension; $\mathrm{AB}=$ abduction; $E R$ = external rotation; 
1 Table 2: Reliability metrics for hip torque measurements using motor-driven dynamometer.

\begin{tabular}{|c|c|c|c|c|c|c|c|}
\hline & Session 1 & Session 2 & ICC (95\%CI) & SEM $(95 \% C I)$ & SEM & MDC (95\%CI) & MDC \\
\hline & $(\mathrm{N} \cdot \mathrm{m})$ & $(\mathrm{N} \cdot \mathrm{m})$ & & $(\mathrm{N} \cdot \mathrm{m})$ & $\%$ & $(\mathrm{~N} \cdot \mathrm{m})$ & $\%$ \\
\hline Abduction $(n=20)$ & $117 \pm 22$ & $113 \pm 25$ & $0.85(0.67-0.94)$ & $9(7-13)$ & 8 & $21(16-31)$ & 18 \\
\hline Adduction $(\mathrm{n}=\mathbf{2 0})$ & $90 \pm 24$ & $95 \pm 27$ & $0.89(0.72-0.95)$ & $8(6-12)$ & 9 & $19(14-28)$ & 20 \\
\hline Extension $(n=19)$ & $152 \pm 27$ & $155 \pm 28$ & $0.95(0.86-0.98)$ & $6(5-10)$ & 4 & $14(11-22)$ & 9 \\
\hline Flexion $(n=20)$ & $97 \pm 22$ & $102 \pm 25$ & $0.95(0.74-0.99)$ & $4(3-7)$ & 4 & $10(7-16)$ & 10 \\
\hline Internal rotation $(n=19)$ & $68 \pm 20$ & $65 \pm 17$ & $0.92(0.78-0.97)$ & $5(4-8)$ & 8 & $12(9-18)$ & 18 \\
\hline $\begin{array}{l}\text { External } \\
(\mathbf{n}=\mathbf{2 0})\end{array}$ & $50 \pm 13$ & $52 \pm 15$ & $0.90(0.75-0.96)$ & $4(3-7)$ & 9 & $10(8-16)$ & 20 \\
\hline
\end{tabular}

2 Values are mean \pm standard deviation unless otherwise indicated; ICC $=$ intraclass correlation coefficient; $\mathrm{CI}=$ confidence interval;

$3 \mathrm{SEM}=$ standard error of measurement $\mathrm{MDC}=$ minimal detectable change; $\mathrm{N} \cdot \mathrm{m}=$ newton.metre $\mathrm{N}=$ newton; $\mathrm{E}=$ extension; $\mathrm{AB}=$

4 abduction; $\mathrm{ER}=$ external rotation; 


\section{Table 3(on next page)}

Reliability metrics for hip force measurements using a custom rig.

Values are mean \pm standard deviation unless otherwise indicated; ICC $=$ intraclass

correlation coefficient; $\mathrm{Cl}=$ confidence interval; SEM = standard error of measurement; MDC

$=$ minimal detectable change; $\mathrm{N}=$ newton; $\mathrm{E}=$ extension; $\mathrm{AB}=$ abduction; $\mathrm{ER}=$ external rotation; 
1 Table 3: Reliability metrics for hip force measurements using a custom rig.

\begin{tabular}{|c|c|c|c|c|c|c|c|}
\hline & Session 1 & Session 2 & ICC (95\%CI) & SEM (95\%CI) & SEM & MDC (95\%CI) & MDC \\
\hline & $(\mathrm{N})$ & $(\mathrm{N})$ & & $(\mathrm{N})$ & $\%$ & $(\mathrm{~N})$ & $\%$ \\
\hline Abduction $(n=20)$ & $120 \pm 26$ & $124 \pm 29$ & $0.77(0.51-0.90)$ & $13(10-19)$ & 11 & $31(26-35)$ & 25 \\
\hline Adduction $(n=20)$ & $158 \pm 33$ & $159 \pm 28$ & $0.89(0.71-0.96)$ & $10(8-16)$ & 7 & $24(23-26)$ & 15 \\
\hline Extension $(n=18)$ & $287 \pm 69$ & $270 \pm 66$ & $0.90(0.73-0.97)$ & $21(16-33)$ & 8 & $49(33-66)$ & 18 \\
\hline Flexion $(n=17)$ & $234 \pm 42$ & $239 \pm 51$ & $0.80(0.53-0.92)$ & $21(16-32)$ & 9 & $49(44-53)$ & 21 \\
\hline Internal rotation $(n=20)$ & $171 \pm 43$ & $169 \pm 39$ & $0.81(0.57-0.92)$ & $18(14-27)$ & 11 & $42(40-43)$ & 25 \\
\hline External rotation $(n=20)$ & $125 \pm 25$ & $130 \pm 29$ & $0.77(0.48-0.91)$ & $13(10-19)$ & 10 & $30(25-35)$ & 23 \\
\hline$E+A B(n=20)$ & $208 \pm 45$ & $207 \pm 52$ & $0.86(0.66-0.95)$ & $19(14-29)$ & 10 & $45(40-50)$ & 23 \\
\hline $\operatorname{E}+\operatorname{ER}(n=20)$ & $211 \pm 54$ & $196 \pm 54$ & $0.86(0.68-0.94)$ & $18(13-27)$ & 9 & $42(41-42)$ & 20 \\
\hline $\mathrm{E}+\mathrm{AB}+\mathrm{ER}(\mathrm{n}=\mathbf{2 0})$ & $201 \pm 55$ & $197 \pm 54$ & $0.88(0.70-0.95)$ & $20(15-29)$ & 10 & $47(32-62)$ & 23 \\
\hline
\end{tabular}

2 Values are mean \pm standard deviation unless otherwise indicated; ICC $=$ intraclass correlation coefficient; $\mathrm{CI}=$ confidence interval;

$3 \mathrm{SEM}=$ standard error of measurement $\mathrm{MDC}=$ minimal detectable change; $\mathrm{N}=$ newton; $\mathrm{E}=$ extension; $\mathrm{AB}=\mathrm{abduction} ; \mathrm{ER}=\mathrm{external}$

4 rotation; 